\title{
Les rapports Orthodoxie/État en Grèce vus par la presse française, 1995-2011
}

Relations between State and Orthodoxy in Greece through the French press, 1995-2011

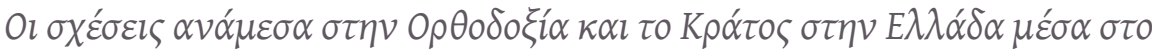

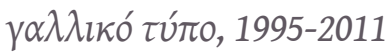

Joëlle Dalègre

\section{(2) OpenEdition} Journals

Édition électronique

URL : https://journals.openedition.org/ceb/3973

DOI : $10.4000 /$ ceb.3973

ISSN : 2261-4184

Éditeur

INALCO

Édition imprimée

Pagination : 209-218

ISBN : 978-2-85831-205-4

ISSN : 0290-7402

Référence électronique

Joëlle Dalègre, «Les rapports Orthodoxie/État en Grèce vus par la presse française, 1995-2011 », Cahiers balkaniques [En ligne], 41 | 2013, mis en ligne le 01 juin 2013, consulté le 06 juillet 2021. URL: http://journals.openedition.org/ceb/3973 ; DOI : https://doi.org/10.4000/ceb.3973

Ce document a été généré automatiquement le 6 juillet 2021

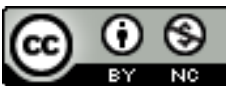

Cahiers balkaniques est mis à disposition selon les termes de la Licence Creative Commons Attribution - Pas d'Utilisation Commerciale 4.0 International. 


\section{Les rapports Orthodoxie/État en Grèce vus par la presse française, 1995-2011}

Relations between State and Orthodoxy in Greece through the French press, 1995-2011

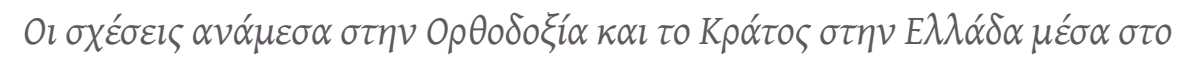

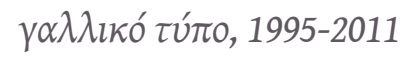

Joëlle Dalègre

1 Ce sujet m'a été inspiré par les fréquentes questions qui m'ont été posées par des personnes qui ne comprenaient pas la situation particulière de la Grèce ou imaginaient un pays de dévots intégristes orthodoxes. J'ai consulté dans les archives du Monde, du Figaro, de La Croix et de Marianne (j'ai abandonné celles de l'Humanité qui ne traitaient que rarement de mon sujet) l'ensemble des articles à partir de 1995 avec des plongées antérieures quand elles étaient révélatrices, l'ensemble - articles, interviews, brèves, communiqués - a représenté 272 textes.

2 Le thème choisi et la visite de Jean-Paul II en terre orthodoxe expliquent à la fois le grand nombre d'articles trouvés dans La Croix (112), la prédominance en nombre de l'année 2001, et les nombreuses signatures de chroniqueurs religieux, en particulier Henri Tincq, l'envoyé à Rome du Monde. J'ai choisi de regrouper ces articles autour de trois thèmes, l'absence de séparation Église/État, le regard très critique porté sur l'orthodoxie, et les doutes exprimés quant à l'appartenance de la Grèce à l'Europe en raison même de son lien à l'orthodoxie.

\section{Un pays qui ignore la séparation Église/État}

3 Tous les journaux, sans exception, mentionnent et répètent régulièrement (en moyenne un texte sur deux) le fait qu'en Grèce il n'y a pas séparation de l'Église et de l'État, ou que l'orthodoxie est reconnue « religion dominante » dans la constitution, les 
autres n'étant que "tolérées ». Le style, le ton laisse toujours comprendre en filigrane (sauf dans Marianne où c'est dit clairement, de manière offensive) qu'exception et étrangeté, ne sont pas des qualités dans ce cas.

\section{On explique le phénomène}

4 Tous citent la forte proportion d'orthodoxes en Grèce, mais, faute de statistiques exactes, le chiffre donné varie de 90 à $98 \%$. Tous également, expliquent et insistent sur le lien historique entre orthodoxie et Grèce, - orthodoxie comme élément de l'identité grecque, comme lien social plus que comme religion -, et sur le lien entre orthodoxie et nationalisme.

Dans une interview au Monde le 21 janvier 1998 Francis Thual déclare :

Dans sa pratique historique, l'orthodoxie slave, grecque, balkanique ou caucasienne n'a jamais connu la laïcité dans sa définition française, fondée sur la séparation de l'Église et de l'État. La séparation du monde confessionnel et de celui de la société civile n'a pas davantage de sens (...) Le national et le religieux se confortent (...) du seul fait de la structuration identitaire de ces sociétés (...). Si l'on veut faire une psychanalyse du monde orthodoxe, sa hantise, c'est la trahison. Il s'agit presque d'une double névrose constituée à la fois par la peur de trahir, et par le sentiment, en tant que dépositaire de la vérité, d'être entouré d'ennemis... C'est un monde martyrisé par l'histoire et empreint en conséquence d'une grande rigidité.

Dans cet esprit, $\mathrm{H}$. Tincq, qui rappelle à plusieurs reprises l'héritage historique, écrit par exemple (Le Monde 16 août 1999) :

Russe, balkanique, byzantine ou arabe, l'orthodoxie est à la fois résistance et martyre (...). Aucune autre religion n'est autant associée au poids des consciences nationales. Aujourd'hui encore, dans les convulsions des Balkans, d'Asie mineure ou centrale, au Proche-Orient, manipulée, instrumentalisée, il lui arrive d'être complice de nationalismes archaïques, de nostalgies d'expansion ou de rêves de restauration. On ne rompt pas aussi facilement avec une histoire qui a commencé par trois siècles de persécutions et par une alliance entre l'Église et un empire qui fut plus souvent synonyme d'asservissement.

7 À propos de cette impossible laïcité, $L a$ Croix, à plusieurs reprises (Le Monde, en un seul article), à l'occasion des discussions sur le fait de savoir si la Constitution européenne doit comprendre dans son introduction une référence à «l'héritage religieux » ou à "l'héritage spirituel», précise que seule la France refuse le terme « héritage religieux », que le mot laikos en grec n'a rien de «laïque » et il énumère les États européens où Dieu figure dans la constitution, ou même comme religion d'État, Irlande, Allemagne, Pologne, Slovaquie, Danemark, Grande-Bretagne, Malte, Albanie, ce qui relativise le cas grec. Le Monde, par la plume d'H. Tincq (12 décembre 2003) parvient à la même conclusion: "Une exception française qui intrigue l'Europe: Ailleurs, l'absence de séparation stricte entre l'État et les Églises n'est pas perçue comme un péril », la Grèce n'est donc pas seule.

\section{On en mesure les conséquences}

8 D'un côté donc, on explique, d'un autre, on s'étonne avec plus ou moins de nuances, de la position matérielle, morale et politique exceptionnelle de l'orthodoxie en Grèce, 
Libération et Marianne sont les plus critiques, quasi-injurieux parfois et énumèrent les « avantages » de l'orthodoxie grecque :

- Une position puissante au point que Marianne titre «la dictature des popes» (11 septembre 2000)

- La première fortune foncière du pays et également d'Israël, un contrôle des banques et de plusieurs entreprises (Marianne, 16 février 2008)

- Des avantages fiscaux

- Un clergé salarié fonctionnaire qui pèse donc sur les dépenses de l'État (Libération 20 mai 2010), et qui aime l'argent : ils demandent 15 euros pour ouvrir la climatisation lors des mariages ou baptêmes! (tous, sauf $L a$ Croix), Marianne, le $1^{\text {er }}$ septembre 2003, titre même " Les popes grecs soignent leur cash-flow ».

- Sa capacité à influencer les élections, à faire reculer un gouvernement, à organiser des manifestations de masse ( 5 articles dans Le Figaro, 14 mentions dans Le Monde) et la conclusion, le 15 mai 2000, de Marianne à propos de la carte d'identité, «la puissance de l'Église orthodoxe est telle qu'elle a réussi ce formidable tour de force : convaincre le pays et ses dirigeants qu'appliquer la loi est illégal et qu’y déroger est légal ! ».

- Une législation à son avantage : enseignement religieux obligatoire à l'école, mention de la religion sur la carte d'identité, autorisation nécessaire du métropolite pour construire n'importe quel bâtiment religieux, serment obligatoire des fonctionnaires. Le Monde, (26 juin 1993) synthétise cette situation :

L'Église est omniprésente dans toutes les sphères de la vie nationale : elle fait partie de la Grèce, elle fait corps avec la grécité, l'hellénisme. Le catéchisme est enseigné à l'école publique, on se marie à l'église même si les socialistes ont instauré en 1983 sans succès apparent le mariage civil. Les entreprises, les bâtiments publics, les habitations, les routes, les navires, les avions sont encensés par les popes. Pas d'inauguration sans prélat. Les ministres, même socialistes, prêtent serment sur la Bible. Les dix mille gens d'Église, véritables fonctionnaires de Dieu, sont payés par le ministère de l'Éducation et des Cultes.

- Sa capacité à intervenir dans les décisions de justice (5 exemples dans Le Figaro dont le 9 septembre 2009, un flash AFP à propos d'une juge condamnée pour avoir, à la demande des moines, trafiqué une décision de justice !

- Les trafics et corruptions, souvent cités dans Le Figaro et dans Libération, stigmatisés dans Marianne (16 février 2008) «une série de turpitudes a éclaboussé les hommes de Dieu: corruption, trafic de drogue, vidéos roses, suicides ", tandis que dans La Croix, on les cite plusieurs fois (8 articles), mais, à chaque fois, pour signaler que la hiérarchie ecclésiastique intervient pour nettoyer ses rangs, invite «à dénoncer les corrompus », tonne contre les mauvais prêtres, les suspend...

\section{L'Athos, l'incarnation des défauts de l'orthodoxie}

9 Tous ces journaux citent les moines du mont Athos, toujours sur un mode ironique, en insistant sur l'interdiction faite aux femmes, et même en souriant (comme Le Monde le $1^{\mathrm{er}}$ octobre 1991) de la mort par le SIDA de 3 moines en 4 ans !

L'apothéose de tous les défauts de l'orthodoxie grecque semble s'incarner dans le mont Athos. Si La Croix ne lui consacre qu'un seul article, son plus long, pour rappeler ses caractères historiques et artistiques, sa valeur religieuse et le recueillement de ses 
moines, à l'inverse, dans les autres journaux, l'aura de l'Athos est bien pâle et même Le Figaro qui le cite dans 19 des 51 textes consultés, en fait le parangon de l'intégrisme et des trafics (10 occurrences). Il s'agit pour lui de la «forteresse orthodoxe" (17 novembre 2000) des «talibans de l'Athos» (15 octobre 2007), qui sont « intouchables » (16 septembre 2008), « pervertis par la manne européenne » (titre du 5 mai 2001) et s'opposent à l'entrée des femmes avec une obstination rétrograde (6 mentions, dont deux articles uniquement sur ce sujet). Tous, sauf La Croix, citent le slogan affiché par des moines contestataires de l'Athos «l'orthodoxie ou la mort », et illustrent cela par la photo d'un pope barbu et hirsute, ainsi dans le Figaro du 4 septembre 2001 où il porte une banderole «the antechrist pope out of orthodox Greece! »

Libération partage cette guerre contre les moines. Sur 33 articles consultés, 10 parlent de l'Athos, sans qu'un seul ne fasse référence à un aspect religieux quelconque. Trois s'indignent contre l'interdiction faite aux femmes, sept traitent des manifestations contre des réformes en désignant les moines de l'Athos comme les éléments moteurs, tous insistent sur leur paradis fiscal, la corruption, les trafics d'antiquité dans lesquels ils sont impliqués, jusqu'à ironiser sur le fait que ces mêmes monastères aient joué, en 1995, des mécanismes européens pour éviter la saisie de leurs terres par l'État. Le long article de Libération « l'île aux ils » (26 août 2008, J B. Harang) réunit toutes les critiques dans un style qui les ridiculise sans pitié, insistant sur les trafics financiers et l'avaton, l'interdiction faite aux femmes :

12 Il existe en Europe occidentale une frontière infranchissable à la moitié du ciel, la moitié de l'humanité. Elle ne sépare pas deux pays, au contraire, elle vient tailler dans le vif de l'un d'eux, amputer la Grèce d'une péninsule sacrée, le mont Athos, au nom de Dieu et au mépris des lois des hommes et des femmes, même s'il s'est toujours trouvé des hommes pour voler au secours des dieux qu'ils inventent et des lois qui les protègent (...) «Pas une femme, pas une chèvre, pas une mule, ânesse ou jument, pour les insectes et les oiseaux on ferme les yeux, pas une bicyclette sans cadre, pas une oie, pas une cane, ni petite ni Grande Ourse, pas la moindre lionne, mais quelques poules qui ne pondent que des œufs mâles, dont le jaune entre dans la composition des pigments des icônes.

\section{On se réjouit du recul progressif des privilèges de l'Église dans l'État}

13 Cependant les rapports entre l'Église et l'État sont présentés comme orageux, car l'Église grecque, même toute puissante, voit ses privilèges rongés par la sécularisation portée par le PASOK, la presse de gauche et une partie de l'opinion. La Croix indique plusieurs fois que les archevêques acceptent des concessions simplement pour éviter une possible séparation Église/État. Mgr Christodoulos le 11 août 2000 (La Croix) a insisté sur le danger à éviter : le gouvernement veut transformer la Grèce « en un État laïque sur le modèle de la France!», expression reprise également par une jeune grecque interviewée (11 septembre 2000 dans Marianne) qui dit de son Premier ministre «Il veut que cela ressemble à la France ».

14 On signale que les reculs sont le résultat d'une obligation européenne ou d'un souci tactique de ne pas pousser le gouvernement à la séparation, dans La Croix, il s'agit aussi 
d'une adaptation obligatoire aux changements apportés en Grèce par la vague d'immigration récente. Parmi les « reculs » notés :

- L'affaire de la carte d'identité sur modèle européen, sans mention de religion, que depuis 1997 la Grèce a acceptée au nom du droit au secret des données personnelles de l'individu, puis refusée, puis acceptée malgré les protestations de l'Église; refusée, acceptée, le feuilleton passionne: 11 articles dans La Croix, 5 dans Le Figaro, 5 dans Marianne, 11 dans Le Monde, 9 dans Libération.

- La fin de l'obligation de l'enseignement religieux dans l'école.

- Le service militaire pour prêtres et moines.

- L'impôt foncier pour les biens d'église conséquence de la confection du cadastre.

- La possibilité de construire un lieu de culte sans autorisation du métropolite et, en particulier le feuilleton de la construction d'une mosquée à Athènes.

-L'autorisation de la crémation des morts

\section{Une religion très critiquée}

15 On se réjouit d'autant plus de ces reculs que la vision de l'orthodoxie est dans l'ensemble très négative, à l'exception de La Croix. Intolérante et antisémite, réactionnaire et intégriste, ultranationaliste (tous ces termes dans Marianne 11 octobre 2000) ; selon ce journal, 11 janvier 2000, la mention de la religion sur la carte d'identité remonterait à l'occupation allemande, visant à repérer les Juifs ; en fait, elle est née de la volonté en 1936 de repérer les communistes.

\section{L'intolérance}

- Le catalogue de plaintes de l'archevêque catholique, Mgr Foscolos, a trouvé écho dans le Monde à plusieurs reprises (16 mars 1995) «le purgatoire des papistes grecs» qui devient « un vrai calvaire » et (4 mai 2001) « un racisme religieux » et, également à 5 reprises dans Le Figaro et autant dans Libération. La Croix, plus complet, signale les difficultés des catholiques, souvent considérés comme des citoyens de second ordre, des évangélistes et des musulmans, l'impossibilité pour eux de devenir officier, d'être enterré dans un cimetière musulman hors de Thrace, l'incendie à deux reprises de la synagogue de La Canée, et précise que, si l'incinération est autorisée, enfin, ce n'est que pour les non-orthodoxes ; il note aussi qu'un métropolite refuse de célébrer les funérailles d'une femme mariée civilement et que l'Église s'oppose toujours à un projet de contrat d'union civile (PACS) (au total 19 mentions à ce sujet dans La Croix).

- Les références aux difficultés des musulmans sont moins évoquées, le plus souvent mises en même temps que celles des catholiques, à l'exception d'une interview mesurée d'un médecin musulman de Thrace Ibram Onsunoglou (Le Monde 8 août 2004) sous le titre «musulman, c'est possible, mais c'est dur ", très bien informé à la fois sur les difficultés et les progrès en cours.

- La visite du pape Jean-Paul II provoquant, un grand nombre d'articles (20 dans La Croix, 14 dans Le Figaro) est l'occasion, pour La Croix et Le Monde, de donner des explications historiques, mais aussi d'insister sur la méfiance, les hésitations ou le mauvais accueil fait par la hiérarchie et les moines à un pape plein de bonne volonté et d'esprit de réconciliation. "Monomanie antipapiste" titre Libération en 1995. On voit le contraste dans le récit du Monde du 6 mai 2001 : 
... d'un côté, Mgr Christodoulos, chef de la puissante Église orthodoxe de Grèce, alignant tous les griefs accumulés contre l'Église romaine; de l'autre côté, la frêle silhouette d'un pape de 81 ans qui a demandé pardon pour les torts du catholicisme romain à l'égard des orthodoxes (...) Mgr Christodoulos a adressé au pape un discours d'une violence qui, partout ailleurs qu'à Athènes, aurait choqué et surpris. Tout y est passé : la «manie destructrice des croisés »; le sac de Constantinople par les Latins ; le "prosélytisme » et l' " activité négative » des " uniates "; les "actes de violence inacceptables » et, même à l'époque moderne, le manque de sensibilité catholique pour les tragédies qui ont frappé la Grèce", et à côté le pape, "Pour toutes les occasions passées et présentes où les fils et filles de l'Église ont péché, par action et par omission, contre les frères et sœurs orthodoxes, puisse le Seigneur nous accorder le pardon que nous lui demandons! »

Récit repris à peu près dans les mêmes termes partout. On voit la bonne volonté du pape, prêt à toutes les concessions, en pays "farouchement orthodoxe", en " orthodoxie hostile » (Le Figaro, 4 mai 2001) face à un clergé qui discute tous les détails de l'ordre du jour, même la musique, Haendel ou Vivaldi ! Là encore seule La Croix est plus consensuelle.

\section{Réactionnaire et intégriste}

- Tous les journaux signalent la condamnation par l'Église du livre de M. Androulakis (en 2000) qui imaginait une liaison entre le Christ et Marie-Madeleine, un texte jugé « injurieux, traître, antipédagogique et produit des frustrations sexuelles de son auteur " (Marianne 20 mars 2000), «La Grèce aussi a son Salman Rushdie », poursuivi également par l'État pour «insulte à la religion ». Libération rappelle à l'occasion, la condamnation de Kazantzakis, tous les journaux, sauf $L a$ Croix, rappellent les condamnations d'un film d'Angelopoulos, du Da Vinci Code ou de La Passion du Christ. L'Église fait également interdire par l'intermédiaire du ministère de la Culture, le culte païen de Zeus prévu par le groupe Ellinais (Le Figaro 15 octobre 2007) à l'Olympeion d'Athènes. Libération (4 janvier 2005) évoque aussi la condamnation d'une BD autrichienne de Gerhard Haderer qui montre un Christ réaliste, clodo drogué, dont les miracles sont des quiproquos dont profite sa secte.

- Si l'actuel archevêque, Hieronymos, est présenté comme un homme prêt aux concessions, l'image de son prédécesseur, Christodoulos, est plus qu'ambiguë : pour $4 / 5$ des journaux, il est populiste et médiatique, plaît aux jeunes, mais se révèle au fond, ultraconservateur et nationaliste (12 fois cité ainsi dans Le Figaro, 10 fois dans Libération, un vrai roi des médias françaises!) Seule La Croix, après plusieurs articles sur son état de santé, le présente comme un homme «qui a fait avancer » les choses, en tentant de trouver un juste milieu entre les moines intégristes et les laïques du PASOK, qui a su ramener des jeunes dans les églises alors même que le recrutement de popes devient plus difficile. Le 2 février 2008 Marianne titre «L'antilaïc rend l'âme » tandis que dans Le Monde : 
Il était vénéré dans les milieux nationalistes, conservateurs, antieuropéens, et détesté par les progressistes et intellectuels. Il incarnait la toute-puissance de l'Église, ultraconservateur, ultranationaliste, flamboyant, populiste, antilaïque " et (31 janvier 2008) Il devient le porte-parole des milieux nationalistes et conservateurs, qualifiant l'homosexualité de "tare», les Turcs de «barbares", moquant les Européens qui n'avaient pas commencé «à créer une civilisation quand les Grecs construisaient le Parthénon ». Il flatte les réflexes antiaméricains, qualifiant les attentats du 11-Septembre de «punition divine».

\section{Quelques nuances}

17 Pourtant quelques nuances existent apportées dans La Croix qui se distingue par son intérêt pour les affaires intérieures à l'orthodoxie et sa vie religieuse.

Une dizaine d'articles présentent les difficultés entre l'Église grecque et le Patriarcat d'Istanbul à propos de la nomination de nouveaux évêques en Macédoine, la lutte de pouvoir entre le Patriarche et Mgr Christodoulos, puis le rapprochement, et insistent également sur la différence entre le Patriarcat totalement opposé aux nationalismes et les églises autocéphales distinctes, idée reprise dans l'un des plus longs articles du Monde, le 20 avril 1994 : «Un entretien avec le patriarche orthodoxe de Constantinople Bartholomée $\mathrm{I}^{\mathrm{er}}$ : Nous condamnons l'exploitation de la religion à des fins nationalistes $»$.

Le Monde publie également, le 5 mai 2001, une interview d'un théologien orthodoxe qui, pour dédouaner Mgr Christodoulos, trouve une argumentation qu'il croit adaptée à un lectorat français :

Ce que refuse le primat de Grèce, MgrChristodoulos, c'est une Europe technocratique qui nierait les identités, une Europe qui ne serait pas fidèle à son héritage biblique, à la défense de valeurs communes et d'une convivialité hors de laquelle on tomberait dans la barbarie. En France, l'homme qui dit non à la mondialisation est forcément un paysan : c'est José Bové. En Grèce, c'est forcément un prêtre, un évêque. Mgr Christodoulos, c'est le José Bové de la Grèce.

La Croix est également le seul à présenter l'action caritative de l'église grecque, renforcée par Mgr Hieronymos qui décida de consacrer à des œuvres supplémentaires l'argent prévu pour la construction d'une nouvelle cathédrale et d'un palais archiépiscopal à Athènes, tout comme l'action en faveur de l'écologie du Patriarcat, rejoint par Mgr Christodoulos qui veut faire "respecter la création de Dieu », "Le monde orthodoxe pionnier de la conscience verte » (20 octobre 2009).

Enfin le même journal signale à plusieurs reprises des rencontres œcuméniques entre chrétiens, entre juifs et orthodoxes et insiste sur le fait que l'orthodoxie n'est pas aussi figée et conservatrice qu'on l'imagine, qu'elle « se renouvelle » (25 juillet 2008), ainsi le 8 février et le 2 mai 2010 « Les 10 défis de l'orthodoxie » puis « La théologie orthodoxe, renouveau $»$.

Dans Le Figaro, deux interviews, celles de Mgr Emmanuel, archevêque orthodoxe de France (15 octobre 2007) et celle d'un théologien orthodoxe, Olivier Clément (4 mai 2001), deux interviews équivalentes dans Le Monde apportent quelques adoucissements à ces vues radicales (2 sur 15 ans !). Une distinction est faite entre les moines, présentés comme le modèle du conservatisme sectaire, les archevêques et le public dont tous les articles précisent qu'il n'est guère pratiquant. 


\section{Une Grèce orthodoxe peut-elle être européenne ?}

Nous sommes Grecs-orthodoxes avant d'être Européens » Mgr Christodoulos en 2000 ( $L a$ Croix). «... Il (un provincial interviewé) énumère les trois piliers de la société grecque : famille, religion, patrie. Une Sainte Trinité que les Hellènes ne sont pas près de troquer contre les lointaines valeurs européennes" ajoute Marianne le 11 septembre 2000.

Les positions de la Grèce face au Kosovo et à la Serbie, puis le différend avec l'Europe sur la carte d'identité, jointes aux déclarations tonitruantes de Mgr Christodoulos qui mettent en jeu l'identité hellénique, ont poussé les commentateurs à affirmer que le lien Grèce/orthodoxie était un obstacle à son "européanisation ", thème récurrent dans la presse (sauf dans La Croix). Selon certains, il semblerait que "Balkans » et «orthodoxie » soient des termes quasi incompatibles avec «Europe» et renvoient directement à "Orient». Tous les différends entre la Grèce et l'UE font également partie des preuves avancées à un moment ou à un autre!

Trois articles ont fait date; l'un, une interview de Jacques Lacarrière, pourtant un grand ami de la Grèce, publiée le 24 novembre 1998 dans Le Monde, les deux autres, écrits par Thierry Desjardins dans Le Figaro, le 24 avril 1999 et le $1^{\text {er }}$ juin 1999. Ils ont été précédés déjà dans Le Monde du 25 juin 1994 des remarques de José Fralon « c'est sur le ton de la plaisanterie que certains hauts responsables communautaires se laissent aller à dire que, "si les Grecs veulent partir, on ne les retiendra pas !"...faisant fi des réalités géopolitiques d'un pays balkanique, gommant la force de l'orthodoxie, niant la fragilité d'un pays sans État, ou presque, les dirigeants de 1979 ont semblé se tromper de partenaire ".

26 Jacques Lacarrière met le feu aux poudres en affirmant entre autres :

Avec l'orthodoxie, c'est (la Grèce) un pays qui est devenu foncièrement balkanique. Il est géographiquement en Méditerranée, mais historiquement, économiquement et spirituellement dans les Balkans et dans le monde orthodoxe, aux côtés de la Russie et de la Serbie. En temps normal, on ne remarque jamais cela, on n'y prête pas attention. Quand on visite la Grèce, on a le sentiment d'être en Europe, une Europe ouverte et humaniste. Mais dès que cela va mal, cet inconscient se fait jour. (...) ils (les Grecs) considèrent que leur avenir se joue davantage à Sofia ou à Belgrade qu'à Bruxelles. Sur le plan économique, juridique et administratif, leur avenir est davantage à Bruxelles. Mais leur sensibilité l'entend autrement, et il n'y a aucun homme politique qui oserait affirmer publiquement que la Grèce doit abandonner complètement les Balkans et s'inscrire dans une perspective purement occidentale. Sa carrière serait terminée.

Enfin, plus grave encore, on lui demande « Pour les Européens du Nord, la Grèce n'estelle pas une sorte de danseuse ? Et il répond : Une danseuse exigeante et coûteuse. Mais on ne peut pas demander à la Grèce d'apporter ce qu'elle n'a pas. Elle ne sera jamais totalement européenne.»

L'année suivante le bilan de Thierry Desjardins est encore plus sévère. Dans « La Grèce, pivot entre deux Europe » (24 avril 1999) il inscrit en sous-titre : «La Grèce fait-elle vraiment partie de l'Europe ? » et il précise plus loin :

Elle est le seul pays de la Communauté à n'avoir aucune frontière avec le reste de l'Union, le seul à avoir un alphabet différent, le seul à être orthodoxe. Elle est donc géographiquement, culturellement, religieusement « en marge». 
oxe de cette Grèce amarrée à l'Europe et à Occident, mais orientale par tous ses pores religieux " écrit Henri Tincq dans Le Monde, peu après que François Thual, interviewé le 20 janvier 1998, ne se soit posé des questions graves de sous-entendus «L'orthodoxie, refuge identitaire, est-elle nécessairement une religion d'État? Peutelle admettre la laïcité ? »... la constitution affirme que "la religion dominante en Grèce est celle de l'Église orthodoxe orientale du Christ. Cela est-il compatible avec l'existence d'un État moderne et avec l'appartenance à l'Union européenne ? ", et plus loin, «Les Grecs sont en Europe, mais se considèrent-ils comme des Européens ? » Les textes laissent suggèrent une réponse négative qu'exprime d'une façon plus agressive le titre de Marianne le 31 juillet 2004, «La Grèce, bastion européen de l'obscurantisme?».

31 Au total, on peut dire que tous les journaux consultés donnent une information assez complète sur le sujet, mais sont très critiques, même quand ils expliquent les phénomènes; cette unanimité, bien différente de ce que l'on peut observer sur d'autres sujets, reflète l'attachement profond à la laïcité. On peut cependant distinguer quelques différences. Libération et Marianne sont les plus critiques, le dernier se distinguant par ses titres provoquants : "La Grèce aussi a son Salman Rushdie », "Bénie soit la carte d'identité ", «La dictature des popes ", "La Grèce, bastion européen de l'obscurantisme?", "Chez les orthodoxes ton prochain tu tueras", «Réveillon orthodoxe : 7 blessés », «L'antilaïc rend l'âme ", «Les popes à l'assaut du Parthénon », «Les popes grecs soignent leur cash-flow", «Je suis orthodoxe, donc je suis" (sur 10/11 titres au total). Les articles les plus longs et les plus complets se trouvent dans Le Monde et dans La Croix, même si expliquer ne signifie pas qu'ils approuvent. Le voyage du Pape a donné la parole aux spécialistes du fait religieux qui ont un ton beaucoup plus empreint de sympathie et nettement moins critique que ceux qui sont rédigés par les correspondants à Athènes, plus préoccupés de la société civile et politique que de religion. L'ensemble reflète néanmoins une laïcité française sûre d'elle, stupéfaite de ce qu'elle découvre, et qui devient même intransigeante quand elle observe la Grèce, comme si on ne pouvait tolérer dans ce pays autrefois tant aimé, ce que l'on tolère chez d'autres. 


\section{RÉSUMÉS}

Comment la presse française présente-t-elle depuis 1995 la présence de l'orthodoxie en Grèce, ses rapports avec l'État? L'étude détaillée de trois quotidiens et d'un hebdomadaire montre que, malgré des positions fort différentes sur d'autres sujets, tous reflètent une incompréhension de base : la France laïque ne comprend pas, ou mal, que l'orthodoxie puisse occuper une aussi large place dans ce pays. Elle détaille donc cette puissance pour la critiquer, se réjouit de voir peu à peu grignoter les privilèges de l'orthodoxie et se demande si on peut être à la fois « orthodoxe » et « européen».

How the French press has presented since 1995 the importance of orthodoxy in Greece and its relations with the State? A detailed study of three daily papers and one weekly magazine shows that, in spite of much different positions on other subjects, all of them reflects the same basic misunderstanding: secular France doesn't understand how Orthodoxy could have a so large place in the country. It details this power to criticize it, rejoices to see its privileges slowly being nibbled and wonders if one may be in the same moment « orthodox » and « European ».

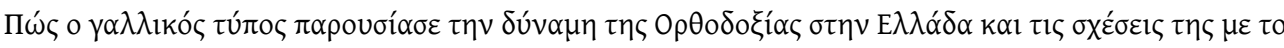

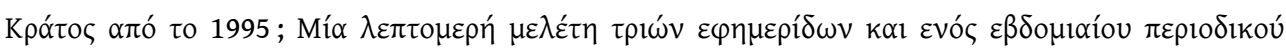

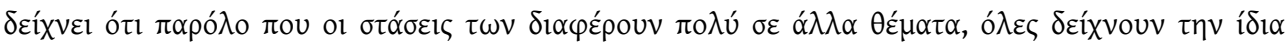

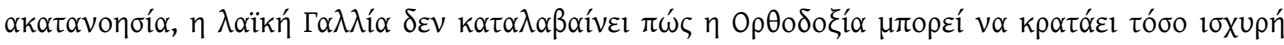

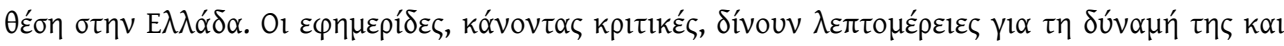

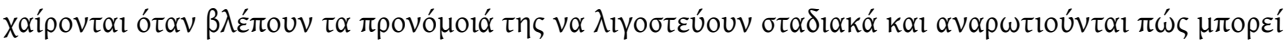

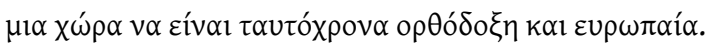

\section{INDEX}

Mots-clés : Avaton/abaton, presse, opinion publique, information, laïcité, rapports Église-État

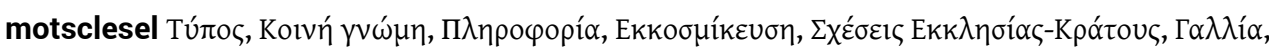

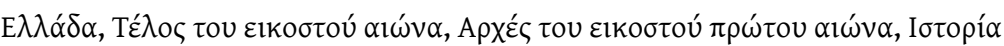

Thèmes : Histoire

motsclesmk ПРИТИСНЕТЕ, МИСЛЕЊЕ ЈАВНОСТА, ИНФОРМАЦИИ, СЕКУЛАРИЗМОТ, ЦРКОВНОДРЖАВА ОДНОСИ, ФРАНЦИЈА, ГРЦИЈА, КРАЈОТ НА ДВАЕСЕТТИОТ ВЕК, ПОЧЕТОКОТ НА ДВАЕСЕТ И ПРВИОТ ВЕК, ИСТОРИЈА

Keywords : press, information, laicity, church-state relations, France, Greece, Beginning of the twenty-first century, History

Index géographique : France, Grèce

motsclestr Basın, Public görüs, Bilgi, Laiklik, Kilise-Devlet İlişkileri, Fransa, Yunanistan, Yirminci yüzyılın sonu, Yirmibirinci yüzyılın başları, Tarih

Index chronologique : vingtième siècle 